\title{
Use of chicken embryonated eggs for evaluating the virulence of Toxoplasma gondii
}

\author{
A. Setasimy $\cdot$ M. Namavari
}

Received: 21 December 2014/ Accepted: 31 January 2015/Published online: 14 February 2015

(C) Indian Society for Parasitology 2015

\begin{abstract}
The objective of the present study was to examine the virulence of Toxoplasma gondii RH strain tachyzoites in embryonated eggs after continuous passage in Vero cell line. The first $T$. gondii tachyzoites was considered low passage (no passage) and then passaged for 80 times on Vero as high passage. Groups of 9-day-old embryonated eggs with ten eggs in each group were inoculated with $10^{2}, 10^{3}$ and $10^{4}$ of low or highpassage $T$. gondii tachyzoites, and any mortality was recorded. Suitable samples from different tissues (liver, heart, and brain) of the dead embryos were collected for histopathological study. In this study, the mortality in group $10^{3}$ and $10^{4}$ was observed, but there was no significant differences in mortality rate in $T$. gondii low passage and high passage. This finding exactly correspond to previous studies that were performed in mice as animal model for $T$. gondii RH strain. Thus on base of this study we could introduce the embryonated eggs as an appropriate animal model to evaluate the virulence of $T$. gondii tachyzoites.
\end{abstract}

Keywords Toxoplasma gondii . Chicken embryonated egg $\cdot$ Virulence $\cdot$ Continuous passage

\section{Introduction}

Toxoplasma gondii is a coccidial and obligate intracellular parasite that infecting all warm-blooded animals, including

\footnotetext{
A. Setasimy

Islamic Azad University Fars Science and Research Branch,

Shiraz, Iran

M. Namavari ( $\square)$

Razi Vaccine and Serum Research Institute, Shiraz, Iran

e-mail: namavari@yahoo.com; m.namavari@rvsri.ac.ir
}

humans (Dubey 2004). If toxoplasmosis acquired in pregnant women, it can cause various congenital infections in the child (Evengård et al. 1999). Cats are the only definitive host and thus the only source of infective oocysts (Ruiz et al. 1973; Dubey 1994), vaccine development to prevent feline oocyst shedding is on progress, mostly with live vaccines, some strain of $T$. gondii are live vaccine that developed for use in sheep (Verma and Khanna 2013). The $\mathrm{RH}$ strain of $T$. gondii is highly virulent and produces lethal infection (Sibley and Boothroyd 1992). Attenuated, temperature-sensitive $T$. gondii strain ts-4 mutant is a suitable candidate for vaccine in order of attenuated virulence (Donald et al. 1996); however, attenuated Toxoplasma has not been done on Vero (African green monkey kidney) cell line. T. gondii can successfully propagated in the embryonated egg and uniform mortality had been observed in many studies (Que et al. 2004; Mello and Deane 1976). Recently, some researchers introduced the embryonated eggs as an animal model for experimental Neospora caninum infection (Khordadmehr et al. 2013; Mansourian et al. 2009; Khodakaram-Tafti et al. 2012) and also for evaluating the virulence of $N$. caninum tachyzoites (Namavari et al. 2012). Since T. gondii and N. caninum are closely related parasites of the phylum Apicomplexa via the comparative genomic (Reid et al. 2012), the aim of this study was to evaluate $T$. gondii virulence by high passage and low passage in Vero cell line using embryonated egg as an animal model.

\section{Materials and methods}

In this investigation, Vero cell lines and $T$. gondii $\mathrm{RH}$ strain were obtained from the Razi Vaccine and Serum Research Institute, Karaj, Iran. Vero cells were maintained in at 
$37{ }^{\circ} \mathrm{C}$ in an atmosphere of $95 \%$ air and $5 \% \mathrm{CO}_{2}$. The tachyzoites of $T$. gondii were cultured on a 24-hour-old monolayer of Vero cell line and maintained in DMEM Medium (Sigma Co., USA) supplemented with $2 \%$ fetal calf serum, penicillin/ml (10,000 U), streptomycin $/ \mathrm{ml}$ $(100 \mu \mathrm{g})$ and streptomycin $(25 \mu \mathrm{g})$ (Invitrogen, USA) at $37{ }^{\circ} \mathrm{C}$ with $5 \% \mathrm{CO}_{2}$ (Hemphill et al. 1996). Tachyzoites were harvested from infected cell cultures when about $80-90 \%$ of the Vero host cells had lysed, as determined by microscopic examination of monolayers for cytopathic effects (CPE), and then counted using a hemacytometer. Viability of the partially purified tachyzoites was evaluated by trypan blue (Gibco) exclusion test (Chamberland and Current 1990). The first $T$. gondii was considered as low passage (no passage) and then passaged for 80 times in vitro (on Vero) as high passage.

Groups of 9-day-old embryonated eggs with ten eggs in each group were inoculated with $10^{2}, 10^{3}$ and $10^{4}$ of lowpassage or high-passage of $T$. gondii tachyzoites on Vero cell line and any mortality was recorded. Suitable samples from various tissues (liver, heart, and brain) of the dead embryos were collected for histopathological and molecular studies. Samples for histopathological study were fixed in $10 \%$ buffer formalin, processed regularly, and embedded in paraffin. Paraffin blocks were then sectioned at about $5 \mu \mathrm{m}$, stained with hematoxylin and eosin (H\&E staining), and studied microscopically with a light microscope.

DNA extraction from 20 to $40 \mathrm{mg}$ of tissues of liver, heart and brain have done using the DNP kit (sina gene co., Iran), according to the manufacturer's instructions. For the detection of parasite DNA, NP6/NP21 primers was used (Burg et al. 1989).

\section{Results}

In the group with $10^{3}$ and $10^{4}$ low passage tachyzoites, eight and ten embryonated eggs died, respectively. In the groups with $10^{3}$ and $10^{4}$ high passage tachyzoites, five and seven embryonated eggs died, respectively (Fig. 1). In groups with $10^{2}$ low passages and either high passage, no mortality was observed. Microscopic examination revealed acute toxoplasmosis in the dead embryos. In the cases with $10^{2}$ tachyzoites, no inflammations were observed in any groups with high or low passage. In other groups granulomatous inflammation was seen with aggregation of numerous macrophages in the liver and heart. In all the point of histopathological changes no differences were seen in different groups of low and high passages. Molecular assay showed the $T$. gondii DNA the embryo liver inoculated with the low and high passage parasite, like in the heart. No parasite DNA were detected in the brain of the dead embryos in any group.

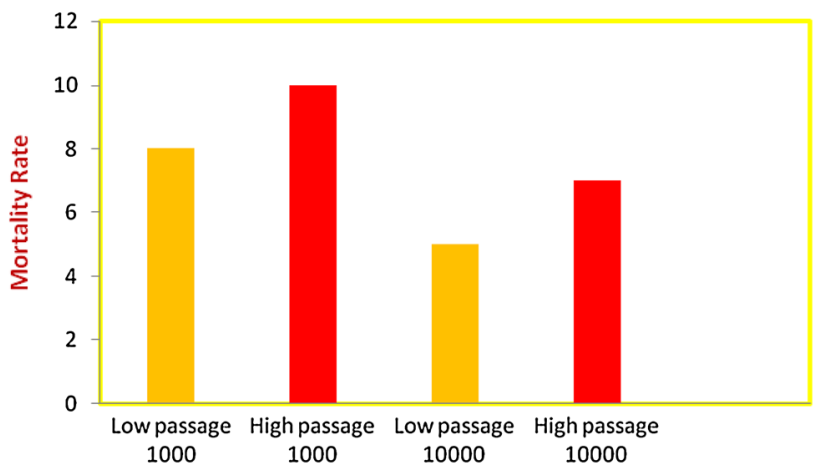

Fig. 1 The mortality rate of chicken embryos inoculated with low and high passage tachyzoite of T. gondii RH strain 103 and 104 inoculum doses

\section{Statistical analysis}

For statistical analysis the Fisher's exact test was used. There was no significant difference between the mortality rate of the low and high passage in all doses of the infected groups with $T$. gondii tachyzoites $(\mathrm{p}<0.05)$.

\section{Discussion}

The development of defensive immunity to $T$. gondii following natural infection in many host species has led researchers to look at vaccination as a strategy to control disease (Innes et al. 2009). Immunization with live, attenuated T. gondii parasites devolved better protection compared to inactivated tachyzoites (Waldeland and Frenkel 1983). Attenuation of parasite virulence has been effectively applied in the development of live vaccines against some protozoan parasites like $N$. caninum (Namavari et al. 2012); Babesia bovis and Babesia bigemina (Pipano et al. 2002). Attenuation of T. gondii virulence has been utilized like the S48 which was maintained in the laboratory by repeated passages in mice (Wilkins et al. 1988); TS-4 isolate which is a chemical mutagenesis of the $\mathrm{RH}$ strain resulted in the temperature sensitive strain (Pfefferkorn and Pfefferkorn 1976).

MacFarlane and Ruchman (1948), Warren and Russ (1948) had introduced the embryonated egg as a good animal model which could enhance successfully and presented appropriate antigen which fixed complement with Toxoplasma immune animal serum.

Besides the availability and affordability of embryonated eggs, the most important reason is the need for a very low LD50 dose of $T$. gondii for infection in the embryonated chicken egg and less incubation time in comparison to the mice. In this study the process of attenuation $T$. gondii virulence was performed by the continuous passage of parasites in the cell culture, which have not lead to 
reduction in virulence. The results of this study indicated course of infection in embryonated egg with $T$. gondii RH strain was independent on the number of passage; therefore the embryonated eggs inoculated with the high-passage tachyzoites compared to those receiving the low-passage tachyzoites have the same mortality rate.

Wilkins et al. (1988) demonstrated T. gondii S48 strain became an attenuated strain following long $(\times 3,000)$ passages in vivo condition. Immunization with attenuated strain reduced neonatal mortality in mice (Buxton et al. 1991); however, Chamberland and Current (1990), showed that continuous passage of $T$. gondii RH grown in mouse macrophage cell lines (in vitro condition) retained virulence, which is confirming with our finding. We conclude on base of this investigation embryonated egg could to be a suitable animal model to evaluating the virulence of $T$. gondii.

Data from previous studies shown that biological and genetical differences between strains of $T$. gondii. As $T$. gondii S48 strain became an attenuated strain following serial passages, in contrast passage in cell-culture over a several months did not affect the pathogenicity of $T$. gondii RH strain in mouse model. In conclusion here we reconfirmed this finding by use of embryonated chicken egg. Consequently we could introduce embryonated chicken egg as suitable animal model to assessment of pathogenicity of different strains of $T$. gondii.

Acknowledgments This study was financially supported by Razi Reference Laboratory of Neospora Research Grant No. 2-84-1891116.

\section{References}

Burg JL, Grover CM, Pouletty P, Boothroyd JC (1989) Direct and sensitive detection of a pathogenic protozoan, Toxoplasma gondii, bypolymerase chain reaction. J Clin Microbiol 8:1787-1792

Buxton D, Thomson K, Maley S, Wright S, Bos HJ (1991) Vaccination of sheep with a live incomplete strain (S48) of Toxoplasma gondii and their immunity to challenge when pregnant. Vet Rec 129:89-93

Chamberland S, Current LW (1990) Use of mouse macrophage cell lines for in vitro propagation of Toxoplasma gondii $\mathrm{RH}$ tachyzoites. Proc Soc Exp Biol Med 2:150-157

Donald RGK, Carter D, Ullman B, Roos DS (1996) Insertional tagging, cloning, and expression of the Toxoplasma gondii hypoxanthine-xanthine-guanine phosphoribosyltransferase gene: use as a selectable marker for stable transformation. J Biol Chem 271:14010

Dubey JP (1994) Toxoplasmosis. J Am Vet Med Assoc 205:1593-1598

Dubey JP (2004) Toxoplasmosis—a waterborne zoonosis. Vet Parasitol 126:57-72

Evengård B, Lilja G, Capraru T, Malm G, Kussofsky E, Oman H et al (1999) A retrospective study of seroconversionagainst
Toxoplasma gondii during 3,000 pregnancies in Stockholm. Scand J Infect Dis 31:127-129

Hemphill A, Gottstein B, Kaufmann H (1996) Adhesion and invasion of bovine endothelial cells by Neospora caninum. Parasitology 112:183-197

Innes EA, Bartley PM, Maley S, Katzer F, Buxton D (2009) Veterinary vaccines against Toxoplasma gondii. Mem Inst Oswaldo Cruz 2:246-251

Khodakaram-Tafti A, Mansourian M, Namavari M, Hosseini A (2012) Immunohistochemical and polymerase chain reactionstudies in Neospora caninum experimentally infected broiler chicken embryonated eggs. Vet Parasitol 188:10-13

Khordadmehr M, Namavari M, Khodakaram-Tafti A, Mansourian A, Rahimian A, Daneshbo Y (2013) Comparison of use of Vero cell line and suspension culture of murine macrophage to attenuation of virulence of Neospora caninum. Res Vet Sci 95:515-521

MacFarlane JO, Ruchman I (1948) Cultivation of Toxoplasma in the developing chick embryo. Exp Biol Med 67:1-4

Mansourian M, Khodakaram-Tafti A, Namavari M (2009) Histopathological and clinical investigations in Neospora caninum experimentally infected broiler chicken embryonated egg. Vet Parasitol 166:185-190

Mello MN, Deane MP (1976) Patterns of development of Trypanosoma cruzi in the embryonated chicken egg. Ann Trop Med Parasitol 70:380-388

Namavari M, Mansourian M, Tafti AK, Hosseini MH, Rahimiyan A, Khordadmehr M, Lotfi M (2012) Application of chicken embryonated eggs as a new model for evaluating the virulence of Neospora caninum tachyzoites. Comp Clin Pathol 21:1665-1668

Pfefferkorn ER, Pfefferkorn LC (1976) Toxoplasma gondii: isolation and preliminary characterization of temperature-sensitive mutants. Exp Parasitol 129:25-34

Pipano E, Shkap V, Kreigel Y, Leibovitz B, Savitsky I, Fish I (2002) Babesia bovis and Babesia bigemina persistence of infection in Friesian cows following vaccination with live antibabesial vaccines. Vet J 164:64-68

Que X, Wunderlich A, Joiner KA, Reed SL (2004) Toxopain-1 is critical for infection in a novel chicken embryo model of congenital toxoplasmosis. Immunol Infect Dis 72:2915-2921

Reid AJ, Vermont SJ, Cotton JA, Harris D, Hill-Cawthorne GA, Ko nen-Waisman S et al (2012) Comparative genomics of the apicomplexan parasites Toxoplasma gondii and Neospora caninum: coccidia differing in host range and transmission strategy. PLoS Pathog 3:e1002567

RuIz A, Frenkel JK, Cerdas L (1973) Isolation of Toxoplasma from soil. J Parasitol 59:204-206

Sibley LD, Boothroyd JC (1992) Virulent strains of Toxoplasma gondii comprise a single clonal lineage. Nature 359:82

Verma R, Khanna Pardeep (2013) Development of Toxoplasma gondii vaccine (A global challenge). Hum Vaccines Immunother 9:291-293

Waldeland H, Frenkel JK (1983) Live and killed vaccines against toxoplasmosis in mice. J Parasitol 69:60-65

Warren J, Russ SB (1948) Cultivation of Toxoplasma in embryonated egg. An antigen derived from choriolallantoic membrane. Exp Biol Med 67:85-89

Wilkins MF, O'Connell E, Te Punga WA (1988) Toxoplasmosis in sheep III.Further evaluation of the ability of a live Toxoplasma gondii vaccine to prevent lamb losses and reduce congenital infection following an experimental oral challenge. NZ Vet $\mathrm{J}$ 36:86-89 